\title{
THE RAPID POINTS OF A COMPLEX OSCILLATION
}

\author{
PAUL POTGIETER
}

Department of Decision Sciences, University of South Africa, P.O. Box 392, Pretoria 0003, South Africa

e-mail address: potgip@gmail.com

\begin{abstract}
By considering a counting-type argument on Brownian sample paths, we prove a result similar to that of Orey and Taylor on the exact Hausdorff dimension of the rapid points of Brownian motion. Because of the nature of the proof we can then apply the concepts to so-called complex oscillations (or algorithmically random Brownian motion), showing that their rapid points have the same dimension.
\end{abstract}

\section{INTRODUCTION}

A popular theme in the study of Brownian motion is the properties and structure of certain compact sets associated with, or generated by, the process. Although this endeavour originally started by examining the Lebesgue measure of such sets, very interesting results were obtained when considering Hausdorff and, subsequently, Fourier dimensions. In this paper, we take the following as our definition of (one dimensional) Brownian Motion:

Definition 1.1. Given a probability space $(\Omega, \mathcal{B}, \mathbf{P})$, a Brownian motion is a stochastic process $X$ from $\Omega \times[0,1]$ to $\mathbb{R}$ satisfying the following properties:

- Each path $X(\omega, \cdot):[0,1] \rightarrow \mathbb{R}$ is almost surely continuous

- $X(\omega, 0)=0$ almost surely

- For $0 \leq t_{1}<t_{2} \cdots<t_{n} \leq 1$, the random variables $X\left(\omega, t_{1}\right), X\left(\omega, t_{2}\right)-X\left(\omega, t_{1}\right), \ldots$, $X\left(\omega, t_{n}\right)-X\left(\omega, t_{n-1}\right)$ are independent and normally distributed with mean 0 and variance $t_{1}, t_{2}-t_{1}, \ldots, t_{n}-t_{n-1}$.

Khinchine's famous law of the iterated logarithm (see, for instance, p67 of [7]) describes the local growth of Brownian motion at almost all points of the unit interval. We state the theorem for completeness. Since the notation will not cause any confusion, we will usually denote the sample path $X(\omega, \cdot):[0,1] \rightarrow \mathbb{R}$ of a Brownian motion simply by $X(\cdot)$.

1998 ACM Subject Classification: G.3, F.1.1.

2000 Mathematics Subject Classification: 68Q30, 60G15, 28A78, $03 \mathrm{H} 05$.

Key words and phrases: Complex oscillations, Brownian motion, rapid points, Hausdorff dimension, nonstandard analysis. 
Theorem 1.2. For $X$ a one dimensional Brownian motion as above, we have that for any prescribed $t_{0}$ on $[0,1]$

$$
\mathbf{P}\left\{\limsup _{h \rightarrow 0} \frac{X\left(t_{0}+h\right)-X\left(t_{0}\right)}{\sqrt{2|h| \log \log 1 /|h|}}=1\right\}=1 .
$$

This implies that almost all points in the unit interval are points of "ordinary" growth. Though of Lebesgue measure 0 , the set of points that violate this growth condition have other fascinating properties. The points of exceptional growth we will consider are known as rapid points.

Definition 1.3. $t$ is called an $\alpha$-rapid point of the sample path $X$ if

$$
\limsup _{h \rightarrow 0} \frac{|X(t+h)-X(t)|}{\sqrt{2|h| \log 1 /|h|}} \geq \alpha .
$$

(We can define the rapid points of any continuous function $f$ analogously by replacing the sample path $X$ with $f$ in the above.)

Orey and Taylor [12 showed that the set of $\alpha$-rapid points has Hausdorff dimension $1-\alpha^{2}$, almost surely, and shortly afterwards Kaufman proved they have equal Fourier dimension [10]. We shall not delve into the theory of Fourier dimension as it relates to stochastic processes here, but the interested reader is referred to [9] for a thorough exploration. The result of Orey and Taylor is very relevant to the investigations of this paper. Section 3 provides an elementary proof of the result, elements of which are used to prove subsequent results on complex oscillations.

There has been much activity recently connecting the theory of descriptive complexity with that of Brownian motion. Complex oscillations were introduced by Asarin and Pokrovskii [1], being functions which are the limit of piecewise linear functions encoded by strings of high Kolmogorov-Chaitin complexity. It was shown that the set $\mathcal{C}$ of complex oscillations, also known as generic Brownian motion, has Wiener measure 1. Fouché [5] proved that there exists a recursive bijection between the set of Kolmogorov-Chaitin random strings (KC-strings, from hereon) and encoded versions of the complex oscillations. The set $\mathcal{C}$ may be considered an effective representation of Brownian motion, since every property which holds almost surely for a Brownian motion also holds for a complex oscillation, provided it has a suitably effective description [4].

Fouché showed in [6] that iterated logarithmic growth is satisfied at all recursive points in $[0,1]$ and posed the question, whether iterated logarithmic growth holds with Lebesgue measure 1. This was answered in the positive by Kjos-Hanssen and Nerode [11. The question now becomes whether Orey and Taylor's result also holds for complex oscillations, which is what we attempt to answer in this paper.

Complex oscillations, or generic Brownian motion, is also known in the literature as algorithmically random Brownian motion. For the purposes of this paper, we shall keep to the term "complex oscillations", as oft repetition favours the shorter form. It is also the author's preference, since it not only refers to the origins of the subject in Kolmogorov complexity, but also distinguishes it from Brownian motion as a distinct, albeit related, phenomenon.

We employ some nonstandard analysis in the next section. The purpose is merely to facilitate the conversion of some standard inequalities into a calculation of the Hausdorff 
dimension of the $\alpha$-rapid points, following the method in [13], without having to resort to more cumbersome covering arguments. It should not be too difficult for readers who do not wish to delve into the nonstandard background to convince themselves that dimension may be calculated by a method of counting intervals.

As mentioned, section 3 provides an elementary proof of the theorem of Orey and Taylor. Although the supporting lemmas were formulated largely to support the main result on complex oscillations, it seemed appropriate to complete the train of thought in this fashion. The advantage of this method of proof is that it lends itself well to more constructive applications, as in the sequel. A concise exposition of the most relevant definitions and results on complex oscillations are presented at the start of section 4. The results of the previous sections are then applied to show that the $\alpha$-rapid points of a complex oscillation do indeed have Hausdorff dimension $1-\alpha^{2}$. The methods used throughout to approximate sets of rapid points are based upon Kaufman's approximations in the paper [10].

\section{A nONSTANDARD FORMUlation of HAUSdorfF Dimension}

Some familiarity with nonstandard analysis is assumed. For a full explanation of the concepts involved, 2] provides an excellent introduction. It is however not necessary to follow this section in order to understand the main results of the paper, since they are formulated (and, for the most part, proved) without reference to nonstandard analysis.

We first consider the standard definition of Hausdorff dimension. Given a compact set $A$ on the unit interval (or any bounded subset of $\mathbb{R}$ ) and $\epsilon>0$, consider all coverings of the set by open intervals $B_{n}$ of lengths smaller than or equal to $\epsilon$. For each cover, form the sum

$$
\sum_{n=0}^{\infty}\left\|B_{n}\right\|^{\alpha},
$$

where $\|\cdot\|$ denotes the length of an interval (i.e., the supremum of the distances between any two points of the set). For each $A$ and $\epsilon>0$, take the infimum over all such sums, as $\left\{B_{n}\right\}$ ranges over all possible covers of $A$ of diameter $\leq \epsilon$ :

$$
S_{\alpha}^{\epsilon}(A)=\inf _{\left\{B_{n}\right\}} \sum_{n}\left\|B_{n}\right\|^{\alpha} .
$$

As $\epsilon$ decreases to $0, S_{\alpha}^{\epsilon}(B)$ increases to a limit meas ${ }_{\alpha}(A)$ (which might be infinite) which is called the $\alpha$-Hausdorff measure of $A$, or the Hausdorff measure of $A$ in dimension $\alpha$.

Definition 2.1. The Hausdorff dimension, $\operatorname{dim} A$, of a compact set $A \subseteq[0,1]$ is the supremum of all the $\alpha \in[0,1]$ for which, for any cover $B$ of $A, \operatorname{meas}_{\alpha}(B)=\infty$. This is equal to the infimum of all $\beta \in[0,1]$ for which there exists a cover $C$ of $A$ such that meas ${ }_{\beta}(C)=0$.

We now consider the interval [0,1], and divide it into $2^{N}$ sections, where $N$ is a hyperfinite (but not finite) natural number. The set $\left\{0, \Delta t, 2 \Delta t, \ldots,\left(2^{N}-1\right) \Delta t\right\}$, where $\Delta t=2^{-N}$, is referred to as the hyperfinite time line with basis $2^{N}$. If $B$ is a subset of the hyperfinite time line, or indeed a subset of the nonstandard reals, we denote by ${ }^{\circ} B$ its standard part. In [13] a nonstandard version of Frostman's lemma is proved, which is used to establish the following: 
Theorem 2.2. Given a compact subset $A$ of $[0,1]$, there is a subset $A_{\mathbf{T}}$ of the hyperfinite time line $\mathbf{T}$ and a hyperfinite number $N \in \mathbb{N} \backslash \mathbb{N}$ such that ${ }^{\circ} A_{\mathbf{T}}=A$ and

$$
\circ\left(\frac{\left|A_{\mathbf{T}}\right|}{N^{\beta}}\right)=\infty \text { for } \beta<\alpha, \quad \circ\left(\frac{\left|A_{\mathbf{T}}\right|}{N^{\beta}}\right)=0 \text { for } \beta>\alpha
$$

if and only if $\operatorname{dim} A=\alpha$.

This result guarantees the existence of a subset of the hyperfinite time line through which Hausdorff dimension can be computed using a counting argument, but the following result shows that any set which satisfies certain properties can be used:

Theorem 2.3. Consider a hyperfinite time line $\mathbf{T}$ based on the hyperfinite number $2^{N}$, for a given $N \in{ }^{*} \backslash \mathbb{N}$. Suppose that a subset $A^{\prime}$ of the time line is such that ${ }^{\circ} A^{\prime}=A$ and for some $\alpha>0$

$$
\circ\left(\frac{\left|A^{\prime}\right|}{2^{N \beta}}\right)>0 \text { for } \beta<\alpha \text { and } \circ\left(\frac{\left|A^{\prime}\right|}{2^{N \beta}}\right)=0 \text { for } \beta>\alpha \text {. }
$$

Then $\alpha=\operatorname{dim} A$.

\section{RAPID POINTS OF BROWNIAN MOTION}

We calculate the dimension of the rapid points in two stages. For the purposes of section 4 , the method will be more important than the results.

Lemma 3.1. If $A$ is the set of $\alpha$-rapid points of $X(t)$, $A$ has a Hausdorff dimension of at most $1-\alpha^{2}$, almost surely.

Proof. We consider a partial covering of $E_{\alpha}$, the set of $\alpha$-rapid intervals of a Brownian motion, by dyadic intervals, the limit superior of which will form a cover of $E_{\alpha}$. Let $n, j \in \mathbb{N}$ and let $\alpha_{1}<\alpha$. We will consider $j$ to be fixed. Define $B_{\alpha_{1}, n}(\omega)$ to be the random set

$$
\begin{array}{r}
\left\{0 \leq k \leq 2^{n}-1: \exists t \in\left[k 2^{-n}, k 2^{-n}+2^{-n-j}\right]\left(2^{n / 2}\left|X\left((k+1) 2^{-n}\right)-X(t)\right|\right.\right. \\
\left.\left.\geq \alpha_{1} \sqrt{2 n \log 2}\right)\right\}
\end{array}
$$

Note that we can either consider these sets as subsets of the integers or as collections of the dyadic intervals these integers represent. Let $A_{\alpha_{1}, n}$ be the event $\left\{\left|B_{\alpha_{1}, n}(\omega)\right| \geq 2^{n\left(1-\alpha_{1}^{2}\right)}\right\}$. The sets of the form $B_{\alpha_{1}, n}(\omega)$ do not form a cover of the rapid points at each stage $n$. However, we can see from continuity that each $\alpha$-rapid point that can be described as the limit of a selection of endpoints of dyadic intervals, as in the construction of the set in 3.1 , will be contained in the limit superior of the $B_{\alpha_{1}, n}(\omega)$. Moreover, the same argument can be made for the rapid points approximable from the left in such a manner, yielding the same bounds on the Hausdorff dimension, hence achieving the desired result for all $\alpha$-rapid points.

We now estimate the probability of $A_{\alpha_{1}, n}$. The distribution of $\left|B_{\alpha_{1}, n}(\omega)\right|$ is binomial and the probability of a success (of a point $t \in[0,1]$ being in $A_{\alpha_{1}, n}$ ) is calculated in [10] to be larger than $2^{-\alpha_{1}^{2} n(1+o(1))}$. We now want to calculate the probability $\mathbf{P}\left(A_{\alpha_{1}, n}\right)$. For this we use an estimate from [3] for the tail of the binomial distribution. If $S_{2^{n}}$ denotes the sum of $2^{n}$ variables which may take value 1 with probability $p$ and 0 with probability $q=1-p$, then we have that

$$
\mathbf{P}\left\{S_{2^{n}} \geq r\right\} \leq \frac{r q}{\left(r-2^{n} p\right)^{2}},
$$


when $r>2^{n} p$. To see that we may use this estimate, note that the requirement implies that we must have $p<2^{-n \alpha_{1}^{2}}$, which is satisfied in this case.

The estimate now becomes

$$
\mathbf{P}\left(A_{\alpha_{1}, n}\right) \leq \frac{2^{n\left(1-\alpha_{1}^{2}\right)}\left(1-2^{\alpha_{1}^{2} n(1+o(1))}\right)}{\left(2^{n\left(1-\alpha_{1}^{2}\right)}-2^{n} p\right)^{2}} .
$$

Not only can some quick calculation show that this term tends to zero as $n$ tends to infinity, but we also have that the sum of all the terms converges, because of the inequalities

$$
\begin{aligned}
\mathbf{P}\left(A_{\alpha_{1}, n}\right) & \leq \frac{2^{n\left(1-\alpha_{1}^{2}\right)}\left(1-2^{n \alpha_{1}^{2}(1+o(1))}\right)}{\left(2^{n\left(1-\alpha_{1}^{2}\right)}-2^{n} p\right)^{2}} \\
& \leq \frac{2^{-n\left(1-\alpha_{1}^{2}\right)}}{\left(2^{n\left(1-\alpha_{1}^{2}\right)}-2^{n} 2^{-\alpha_{1}^{2}(1+o(1)) n}\right)^{2}} \\
& \leq \frac{1}{2^{\left(1-\alpha_{1}^{2}\right) n}\left(1-2^{-\alpha_{1}^{2} o(1)}\right)^{2}} \\
& \leq \frac{1}{2^{n\left(1-\alpha_{1}^{2}\right)}\left(1-2^{2 \alpha_{1}^{2}(n-1)}\right)} \text { since } 2^{-n \alpha_{1}^{2}(1+o(1))}<1 \\
& \leq \frac{1}{2^{n\left(1-\alpha_{1}^{2}\right)} 2^{2 \alpha_{1}^{2}(n-1)}} \leq \frac{4}{2^{n\left(1+\alpha_{1}^{2}\right)}},
\end{aligned}
$$

where we assume $n$ is large enough so that $r-2^{n} 2^{-\alpha_{1} 2(1+o(1))}>0$. Seeing the above as the first step in constructing our cover, we can now proceed to larger values of $n$ and $\alpha_{1}$ to find intervals of smaller diameter. For such larger values the above inequalities will still hold. We also consider, for each larger value of $n$, a larger value $\alpha_{i}$, where $\alpha_{1}<\alpha_{i-1}<\alpha_{i}<\alpha$. If we now consider, for a specific sequence $\left\{\alpha_{i}\right\}_{i \in \mathbb{N}}$, the collection of intervals given by all the $B_{\alpha_{i}, n}$, we obtain a cover of $A$. Although we have constructed the sets as unions of closed intervals, they may as well be considered to be made up of open intervals, since the set of dyadic rationals has Hausdorff dimension 0. Although the compactness of the set ensures that we could find a finite subcover, we do not actually need to find such a cover here, since the number of intervals used is small enough. If we now consider the $1-\alpha_{1}^{2}$-Hausdorff sum for the cover of $A$ obtained by the above process, we get an expression smaller than

$$
\sum\left|B_{\alpha_{i}, n}\right| 2^{-\left(1-\alpha_{1}^{2}\right) n}<\sum 2^{n\left(1-\alpha_{i}^{2}\right)} 2^{-\left(1-\alpha_{1}^{2}\right) n}=\sum 2^{\left(\alpha_{1}^{2}-\alpha_{i}^{2}\right) n}
$$

which is bounded, as long as we have chosen, for instance, $\alpha_{i}>\left(\alpha+\alpha_{1}\right) / 2$ for all $i \geq 2$. Since the above sum is clearly larger than that of any $1-\alpha_{1}^{2}$-Hausdorff sum for any dyadic cover of the lim sup of the sets $A_{\alpha_{i}, n}$, we have that such Hausdorff sums are bounded for any $\alpha_{1}<\alpha$. The lim sup of $A_{\alpha_{i}, n}$ describes the event that there are more than $2^{1-\alpha_{i}^{2}}$ rapid intervals for arbitrarily large $n$; by the first Borel-Cantelli lemma this has measure 0 , since the probabilities calculated above converge. Hence, with probability 1, we can find for each $\alpha_{1}<\alpha$ a cover of the $\alpha$-rapid points for which the $1-\alpha^{2}$-Hausdorff sum converges, implying a Hausdorff dimension of at most $1-\alpha^{2}$.

We now turn to a requirement which will allow certain sets to have a dimension of no less than $1-\alpha^{2}$ :

Lemma 3.2. Suppose $0<\alpha<1$. Let $f$ be a continuous function and consider an equipartition of $[0,1]$ into $2^{n}$ intervals, each of which is further subdivided in a further $2^{j}$ equal 
pieces. If there exists some $c>0$, dependent only on $f$, such that the relation

$$
\begin{array}{r}
\mid\left\{0 \leq k \leq 2^{n}-1: \exists t \in\left[k 2^{-n}, k 2^{-n}+2^{-n-j}\right]\left(2^{n / 2}\left|f\left((k+1) 2^{-n}\right)-f(t)\right|\right.\right. \\
\geq \alpha \sqrt{2 n \log 2}\} \mid) \geq c 2^{\left(1-\alpha^{2}-\varepsilon\right) n}
\end{array}
$$

is satisfied for arbitrarily large $n$ and arbitrarily small $\varepsilon$, the $\alpha$-rapid points of $f$ have dimension larger than or equal to $1-\alpha^{2}$.

Proof. Consider the relation

$$
\begin{array}{r}
\forall m \in \mathbb{N} \exists n \geq m \forall \varepsilon>0 \mid\left\{0 \leq k \leq 2^{n}-1: \exists t \in\left[k 2^{-n}, k 2^{-n}+2^{-n-j}\right]\right. \\
\left.\left(2^{n / 2}\left|f\left((k+1) 2^{-n}\right)-f(t)\right|\right) \geq \alpha \sqrt{2 n \log 2}\right\} \mid \geq c 2^{\left(1-\alpha^{2}-\varepsilon\right) n} .
\end{array}
$$

Everything in this relation is first order and can be transferred to a hyperfinite context; it follows that

$$
\begin{aligned}
& \forall M \in{ }^{*} \mathbb{N} \exists N \geq M \forall \epsilon>0 \mid\left\{1 \leq K \leq 2^{N}-1: \exists T \in\left[K 2^{-N}, K 2^{-N}+2^{-N-j}\right]\right. \\
& \left.\left.2^{N / 2} \mid F\left((K+1) 2^{-N}\right)-F(T)\right\} \mid \geq \alpha \sqrt{2 N \log 2}\right\} \mid \geq c 2^{\left(1-\alpha^{2}-\epsilon\right) N} .
\end{aligned}
$$

( $F$ is an S-continuous nonstandard lifting of $f$; see for instance [2] or [13].) Now, instead of seeing the division of $[0,1]$ as an equipartition, we can consider it a hyperfinite time line. Also, remembering the ultrapower construction, each $K$ for which the above holds implies the existence of a sequence of dyadic rationals which converges to a rapid point. The hyperfinite dyadic rationals included in the transferred relation therefore exist in the monad (infinitesimal neighbourhood) of an $\alpha$-rapid point. The set of such hyperfinite rationals therefore forms an internal subset of the time line, whose real part is contained in the set of $\alpha$-rapid points of Brownian motion. Let this nonstandard set be denoted by $E_{\alpha}^{\prime}$. We know that there are $\geq c 2^{\left(1-\alpha^{2}-\epsilon\right) N}$ points on our time line of $2^{N}$ elements. To let the quotient

$$
\frac{\left|E_{\alpha}^{\prime}\right|}{2^{N \beta}}
$$

therefore have real part $0,2^{N}$ would have to be raised to a power of at least $1-\alpha^{2}-\epsilon$, for each $\epsilon>0$. Thus, $\operatorname{dim} E_{\alpha} \geq 1-\alpha^{2}$.

We now confirm that Brownian motion does indeed satisfy the previous lemma almost surely, asymptotically.

Lemma 3.3. Given $0<\alpha<1$, there exists a constant $c<1$ such that Brownian motion satisfies relation (3.3) with probability tending to 1 as $n \rightarrow \infty$; that is,

$$
\begin{aligned}
\forall m \in & \mathbb{N} \exists n \geq m \forall \varepsilon>0 \mid\left\{0 \leq k \leq b 2^{n}-1: \exists t \in\left[k 2^{-n}, k 2^{-n}+2^{-j}\right]\right. \\
& \left(2^{n / 2}\left|X\left((k+1) 2^{-n}\right)-X(t)\right| \geq \alpha \sqrt{2 n \log 2}\right\} \mid \geq c 2^{\left(1-\alpha^{2}-\epsilon\right) n} .
\end{aligned}
$$

Proof. We again use a binomial distribution on the set of intervals, viewing it as a Bernoulli trial with probability of success $p$ (as previously used). Using essentially the same estimate of the binomial tail (but for $r$ failures instead of successes) from [3], we now must satisfy requirement of $r<2^{n} p$.

The probability of being an $\alpha$-rapid interval of length $h$ can be bounded from below by the probability of the maximum over the unit interval being larger than $\alpha \sqrt{2 h \log h^{-1}}$, which in turn (by the reflection principle, see, for instance, p26 of [8]) is twice the probability of $X(1)$ being larger than $\alpha \sqrt{2 h \log h^{-1}}$. 
Using the approximation [7]

$$
\left(\frac{1}{y}-\frac{1}{y^{3}}\right) e^{-\frac{1}{2} y^{2}} \leq \frac{1}{\sqrt{2 \pi}} \int_{y}^{\infty} e^{-\frac{x^{2}}{2}} d x
$$

we find that $p>2^{-\alpha^{2} n} \alpha^{-1}\left(2 \log h^{-1}\right)^{-\frac{1}{2}}$. Now, given $r=2^{n\left(1-\alpha^{2}-\varepsilon\right)}$, it is easily verified that $r<2^{n} p$ for large $n$. The approximation of the binomial distribution then yields

$$
\begin{aligned}
\mathbf{P}\left\{S_{m} \leq r\right\} & \leq \frac{(m-r) p}{(m p-r)^{2}} \\
& \leq \frac{\left(2^{n}-2^{\left(1-\alpha^{2}-\varepsilon\right) n}\right) 2^{-\alpha^{2} n}}{\left(2^{n} 2^{-n \alpha^{2}(1+o(1))}-2^{\left(1-\alpha^{2}-\varepsilon\right) n}\right)^{2}} \\
& \leq \frac{1-2^{-\left(\alpha^{2}+\varepsilon\right) n}}{2^{n\left(1-\alpha^{2}\right)}\left(2^{-n \alpha^{2} o(1)}-2^{-\varepsilon n}\right)^{2}} \\
& =\frac{2^{\left(\alpha^{2} o(1)+\varepsilon\right) n}}{2^{n\left(1-\alpha^{2}\right)}\left(2^{\varepsilon n}-2^{\alpha^{2} n o(1)}\right)^{2}}
\end{aligned}
$$

Since $\varepsilon>0$ is fixed in this case, we take $n$ to be large enough so that the second factor of the denominator is $>1$ and also $\alpha^{2} o(1)<\varepsilon$. The approximation then becomes smaller than $2^{-n\left(1-\alpha^{2}-2 \varepsilon\right)}$. This clearly tends to 0 and thus the probability of more than $2^{\left(1-\alpha^{2}-\varepsilon\right) n}$ successes in $2^{n}$ trials goes to 1 .

It now follows trivially from the previous two lemmas that the $\alpha$-rapid points of a Brownian motion have a Hausdorff dimension of $1-\alpha^{2}$, almost surely.

This theorem has the following famous result as a simple consequence [12]:

Corollary 3.4. For a Brownian path $X$,

with probability 1 .

$$
\operatorname{dim}\left\{t: \limsup _{h \rightarrow o} \frac{X(t+h)-X(t)}{\left(2 h \log \log h^{-1}\right)^{\frac{1}{2}}}=\infty\right\}=1
$$

Proof. It is easily seen that that for each $\alpha$, the set of $\alpha$-rapid points has the property of the above set, with probability 1 (the iterated logarithm is too weak to "contain" the growth at the rapid points). The above set therefore contains all the $E(\alpha)$ and has dimension 1, with probability 1.

In the next section we will repeatedly use the probability that a section contains an $\alpha$-rapid point, approximated by $h^{\alpha^{2}} h^{o(1)}$, where $h$ is the length of the interval. This is very close to our approximation of the ratio of intervals which are picked at any stage.

\section{Complex oscillations}

In this part of the paper we consider the descriptive complexity of Brownian motion. A thorough treatment of this topic can be found in [4. After a brief introduction to the concepts, we show how some of the above results also hold for complex oscillations. Throughout we stick close to the original notation and formulation by Fouché. 
P. POTGIETER

4.1. Kolmogorov complexity and complex oscillations. We denote the set of nonnegative integers by $\omega$, and the product space $\{-1,1\}^{\omega}$ by $\mathcal{N}$. The set of words over the alphabet $\{-1,1\}$ is denoted by $\{-1,1\}^{*}$. The usual notation $\sum_{r}^{0}, \prod_{r}^{0}$ and $\Delta_{r}^{0}$ is used to indicate the arithmetical subsets of $\omega^{k} \times \mathcal{N}^{l}, k, l \geq 0$. Lebesgue measure is denoted by $\lambda$ and the set of Kolmogorov-Chaitin binary strings by $K C$. The Kolmogorov complexity of a word $\alpha$ is denoted by $K(\alpha)$.

A sequence $\left(a_{n}\right)$ of real numbers is said to converge effectively to 0 if for some total recursive function $f: \omega \rightarrow \omega$, we will have that $\left|a_{n}\right| \leq(m+1)^{-1}$ when $n \geq f(m)$, for all $n, m<\omega$. A subset $A$ of $\mathcal{N}$ is of constructive measure 0 if there is a recursive function $\phi: \omega^{2} \rightarrow\{-1,1\}^{*}$ such that $A \subset \cap_{n} \cup_{m}[\phi(n, m)]$, where $\lambda\left(\cup_{n}[\phi(n, m)]\right)$ converges effectively to 0 as $n \rightarrow \infty$. Equivalently, $A$ is of constructive measure 0 if there is a $\sum_{1}^{0}$ predicate $P$ such that, if we define $A_{n} \subset \mathcal{N}$ by

$$
\alpha \in A_{n} \Longleftrightarrow \exists k P(n, \bar{\alpha}(k)),
$$

then $A \subset \cap_{n} A_{n}$, and moreover, $\lambda\left(A_{n}\right) \rightarrow 0$ effectively as $\rightarrow \infty$.

We first consider Asarin and Pokrovskii's definition of complex oscillations.

For $n \geq 1$, we write $C_{n}$ for the class of continuous functions on $[0,1]$ that vanish at 0 and are piecewise linear with slope $\pm \sqrt{n}$ on the intervals $[(i-1) / n, i / n], i=1, \ldots, n$. One can associate a binary string $a_{1} \ldots a_{n}$ to every $x \in C_{n}$ by setting $a_{i}=1$ or $a_{i}=-1$ according to whether $x$ increases or decreases on the interval $[(i-1) / n, i / n]$. We call the word $a_{1} \ldots a_{n}$ the code of $x$ and denote it by $c(x)$. Conversely, every binary string $s$ of length $n$ clearly defines a unique element of $C_{n}$. The associated function is denoted by $\psi(s)$. We call a sequence $\left(x_{n}\right)$ in $C[0,1]$ complex if $x_{n} \in C_{n}$ for each $n$ and there is a constant $d$ such that $K\left(c\left(x_{n}\right)\right) \geq n-d$ for all $n$. A function $x \in C[0,1]$ is a complex oscillation if there is a complex sequence $\left(x_{n}\right)$ such that $\left\|x_{n}-x\right\|$ converges effectively to 0 (in the uniform norm) as $n \rightarrow \infty$. The following is a fundamental result in the theory of complex oscillations [1]:

Theorem 4.1. A continuous function on the unit interval is almost surely, with respect to Wiener measure, a complex oscillation.

The theorem further yields information on the rate of convergence, but that will not be germane to our investigation. We now discuss the fundamental tool in the study of complex oscillations.

4.2. Effective generating sequences. In order to recursively characterise almost sure events (with respect to Wiener measure) which are reflected in each complex oscillation, we use an analogue of a $\prod_{2}^{0}$ subset of $C[0,1]$ of constructive measure 0 [4].

We first introduce some notation, maintaining consistency with [5] throughout. If $F$ is a subset of $C[0,1]$, we denote by $\bar{F}$ the topological closure of $F$ in $C[0,1]$. For $\varepsilon>0$ we let $O_{\varepsilon}(F)$ be the set $\left\{f \in C[0,1]: \exists_{f \in F}\|f-g\|<\varepsilon\right\}$. In the sequel, the complement of $F$ is denoted by $F^{0}$ and $F$ by $F^{1}$.

Definition 4.2. (Fouché [5]) A sequence $\mathcal{F}_{0}=\left(F_{i}: i<\omega\right)$ in $\Sigma$ (the Borel subsets of $C[0,1])$ is an effective generating sequence if

1. for $F \in \mathcal{F}_{0}, \varepsilon>0$ and $\delta \in\{0,1\}$, we have, for $G=O_{\varepsilon}\left(F^{\delta}\right)$ or $G=F^{\delta}$, that $W(\bar{G})=$ $W(G)$; 
2. there is an effective procedure that yields, for each sequence $0 \leq i_{1}<\cdots<i_{n}<\omega$ and $k<\omega$, a binary rational number $\beta_{k}$ such that

$$
\left|W\left(F_{i_{1}} \cap \cdots \cap F_{i_{n}}\right)-\beta_{k}\right|<2^{-k} ;
$$

3. for $n, i<\omega$, a strictly positive rational number $\varepsilon$ and $x \in C_{n}$, both the relations $x \in$ $O_{\varepsilon}\left(F_{i}\right)$ and $x \in O_{\varepsilon}\left(F_{i}^{0}\right)$ are recursive in $x, \varepsilon, i$ and $n$.

Given an effective generating sequence $\left(\right.$ EGS) $\mathcal{F}_{0}$, the algebra $\mathcal{F}$ it generates can be effectively enumerated as a sequence of finite intersections of elements of the EGS or their complements. $\mathcal{F}$ is referred to as the effectively generated algebra generated by $\mathcal{F}_{0}$. For a total recursive function $\phi: \omega \rightarrow \omega$ and some effective enumeration $\left(T_{i}\right)$ of $\mathcal{F}$, we say the sequence $\left(T_{\phi(n)}\right)$ is $\mathcal{F}$-semi-recursive. The union of an $\mathcal{F}$-semi-recursive sequence over all $n$ is termed a $\sum_{1}^{0}(\mathcal{F})$ set. The complement of a $\sum_{1}^{0}(\mathcal{F})$ set is called a $\prod_{1}^{0}(\mathcal{F})$ set. If for a sequence $\left(B_{n}\right)$ of sets in $\mathcal{F}$ there exists a total recursive function $\phi: \omega^{2} \rightarrow \omega$ and an effective enumeration $\left(T_{i}\right)$ of $\mathcal{F}$ such that each $B_{n}$ can be described as $\bigcup_{m} T_{\phi(n, m)}$, it is called a uniform sequence of $\sum_{1}^{0}(\mathcal{F})$ sets. The intersection of such a sequence of sets is called a $\prod_{2}^{0}(\mathcal{F})$ set.

Theorem 4.3. [4] Let $\mathcal{F}$ be an effectively generated algebra of sets. If $x$ is a complex oscillation, then $x$ is in the complement of every $\prod_{2}^{0}(\mathcal{F})$ set of constructive measure 0.

The next theorem is also important for our purposes:

Theorem 4.4. [4] If $\left(A_{k}\right)$ is a uniform sequence of $\Sigma_{1}^{0}(\mathcal{F})$ sets with $\sum_{k} W\left(A_{k}\right)<\infty$, then, for each complex oscillation $x$, it is the case that $x \notin A_{k}$ for all large values of $k$.

4.3. The rapid points of complex oscillations. We now state the main theorem of the paper.

Theorem 4.5. The $\alpha$-rapid points of any complex oscillation have Hausdorff dimension $1-\alpha^{2}$.

Proof. In order to effectively describe the rapid points, we adapt an effective generating sequence used in [4. The proof that this is actually an EGS proceeds analogously to the proof in [4, and it would be redundant to reproduce here.

We denote by $[M(I) \leq b]$ the event $[\sup \{X(t): t \in I\} \leq b]$. For $I$ a dyadic subinterval of $[0,1]$ and $b$ a computable real number, we consider the events $[M(I) \leq b]$. Given a specific interval of the form $\left[i 2^{-k},(i+1) 2^{-k}\right]$, we can form a new Brownian motion $Y_{k, i}(t)=$ $X(t)-X\left(i 2^{-k}\right)$. Now let $\left[M_{k, i}(I) \leq b\right]$ be the event $\left[\sup \left\{Y_{k, i}(t): t \in\left[(i+1) 2^{-k}-2^{-j},(i+\right.\right.\right.$ $\left.\left.\left.1) 2^{-k}\right]\right\} \leq b\right]$ ( $j$ is fixed throughout). We use such sets to form our effective generating sequence. Although we now consider the rapid points that are approximated from the left by a dyadic sequence, by the symmetry aspects of Brownian motion, this has the same Hausdorff dimension as the ones that can be approximated from the right. Computing the dimension of either will yield the result. Since any rapid point can be approximated as one or the other (or both), this is sufficient.

In order to do so, we must be able to effectively enumerate them (from the argument in [4, this will suffice). Since at each stage $k$ the number of processes $Y_{k, i}$ is finite, we only need to effectively enumerate the right-hand side of the inequalities. Thus, we consider all $b$ of the form $\beta 2^{-\frac{k}{2}} \sqrt{2 k \log 2}$ for some fixed rational $\beta \leq \alpha$. The EGS formed by such sets 
is denoted by $\mathcal{F}_{0}$ and the algebra which it generates by $\mathcal{F}$. Our aim is henceforth to use this algebra to effectively describe the event of a complex oscillation having more or less than a certain number of rapid intervals, and hence to find approximations of the Hausdorff dimension in the sense of Lemmas 3.1 and 3.2 .

Firstly we show that a complex oscillation must have at least a certain number of $\beta, n$-rapid intervals for large $n$. To do so, we consider the number of possible choices of $\left\lfloor 2^{\left(1-\beta^{2}-\varepsilon\right) n}\right\rfloor$ intervals out of the $2^{n}$ total intervals at stage $n$, where $\varepsilon$ is a small rational.

Let the set of possible choices of this many intervals per stage $n$ be denoted by $\mathfrak{C}_{n}$, which clearly has cardinality

$$
\left(\begin{array}{c}
2^{n} \\
\left\lfloor 2^{n\left(1-\alpha^{2}-\varepsilon\right)}\right\rfloor
\end{array}\right)
$$

Let $\left\{C_{n, i}\right\}$ denote the $i$-th choice of intervals in some ordering (e.g. lexicographic) of $\mathfrak{C}_{n}$.

Consider the events

$$
B_{\beta, n, i}=\bigcap_{I \in C_{n, i}}\left[\sup \left\{\left|Y_{k, i}(t)\right|: t \in I\right\} \geq \beta 2^{n / 2} \sqrt{2 n \log 2}\right] .
$$

Thus, each $B_{\beta, n, i}$ is an effective finite conjunction of elements of the EGS and hence in the algebra $\mathcal{F}$ describing the event of all intervals in $C_{n, i}$ being rapid.

We want to show that, for a complex oscillation $x$, it is impossible for $x$ not to be contained in some $B_{\beta, n, i}$ for large $n$. To do so, we consider the event of always being able to find a collection of more than $2^{n}-\left\lfloor 2^{\left(1-\beta^{2}-\varepsilon\right) n}\right\rfloor$ intervals which are non-rapid - since in such a case it is impossible to find enough rapid intervals. Hence, let the number of possible choices of $2^{n}-\left\lfloor 2^{\left(1-\beta^{2}-\varepsilon\right) n}\right\rfloor$ intervals out of $2^{n}$ be effectively enumerated at each stage $n$ and call the $i$ th member of the collection $D_{n, i}$. Let

$$
A_{\beta, n, i}=\bigcap_{I \in D_{n, i}}\left[\sup \left\{\left|Y_{k, i}(t)\right|: t \in I\right\}<\beta 2^{n / 2} \sqrt{2 n \log 2}\right],
$$

which therefore describes the event of all intervals in $D_{n, i}$ being non-rapid. Since each of the $A_{\beta, n, i}$ can be described as an effective conjunction of elements of the algebra, we can find a recursive function $\psi: \omega \times \omega \rightarrow \omega$ for an effective enumeration $\left\{T_{i}\right\}$ of the algebra (with $T_{0}$ assumed to be $\emptyset$ ) such that $A_{\beta, n, i}=T_{\psi(n, i)}$. The function $\psi$ is however not total, but can easily be extended to be such by setting $\psi(n, i)=0$ for $i \in \omega$ previously undefined. We form the sets

$$
A_{\beta, n}=\bigcup_{i} T_{\psi(n, i)}
$$

which then form a uniform sequence of $\Sigma_{1}^{0}(\mathcal{F})$ sets. If we can show that $\sum_{n} W\left(A_{n}\right)<\infty$, we know that for any $x \in \mathcal{C}, x \notin A_{k}$ for large $k$. This would mean that for each $x \in \mathcal{C}$, we are unable to choose more than $2^{n}-\left\lfloor 2^{\left(1-\beta^{2}-\varepsilon\right) n}\right\rfloor$ non-rapid intervals, implying that there must be $\left\lfloor 2^{\left(1-\beta^{2}-\varepsilon\right) n}\right\rfloor$ rapid intervals. But we have shown in the proof of Lemma 3.3 that the probability of such an event $A_{\beta, n}$ for each $n$ is less than $1 / 2^{\left(1-\beta^{2}-2 \varepsilon\right) n}$, which completes the argument.

We now use a similar argument to show that there cannot eventually be more than $\left\lceil 2^{\left(1-\beta^{2}\right) n}\right\rceil$ rapid intervals out of $2^{n}$. We look at possible choices of $\left\lceil 2^{\left(1-\beta^{2}\right) n-1}\right\rceil$ dyadic intervals out of $2^{n}$ and denote the effective numbering of these by $C_{n, i}^{\prime}$. We consider, for each $n$, the event

$$
\exists i \text { (All intervals in } C_{n, i}^{\prime} \text { are } \beta, n \text {-rapid) . }
$$


Similarly to the previous, this forms a uniform sequence of $\Sigma_{1}^{0}(\mathcal{F})$ sets. According to the proof of Lemma 3.2, the sum of these sets over $n$ once again converges, implying that, for large $k$, no complex oscillation can have more dyadic rapid intervals than $2^{\left(1-\beta^{2}\right) n}$.

Since these estimates will hold for any $\beta<\alpha$, we can conclude that the $\alpha$-rapid intervals have Hausdorff dimension $1-\alpha^{2}$.

\section{ACKNOWLEDGEMENT}

The author would like to thank Willem Fouché for not only introducing him to the subject, but also for his professional guidance and helpful remarks in the preparation of this paper.

\section{REFERENCES}

[1] Asarin, E.A. and Pokrovskii, A.V. Use of the Kolmogorov complexity in analyzing control system dynamics. Automation and Remote Control, 47:21-28, 1986.

[2] Cutland, N.J. Loeb Measures in Practice: Recent Advances. Springer-Verlag, 1997.

[3] Feller, W. An Introduction to Probability Theory and Its Applications Vol. 1, 3rd (revised) ed. Wiley, 1970.

[4] Fouché, W.L. Arithmetical representations of Brownian motion I. J. Symbolic Logic, 65:421-442, 2000.

[5] Fouché, W.L. The Descriptive Complexity of Brownian Motion. Advances in Mathematics, 155(2):317343, 2000.

[6] Fouché, W.L. Dynamics of a generic Brownian motion: Recursive aspects. Theoretical Computer Science, 394(3):175-186, 2008.

[7] Freedman, D. Brownian motion and diffusion. Holden-Day San Francisco, 1971

[8] K. Itô and H.P. McKean, Jr. Diffusion Processes. Springer-Verlag, 1974.

[9] Kahane, J-P.: Some Random Series of Functions, 2nd ed. Cambridge University Press, 1985

[10] Kaufman, R. Large increments of Brownian motion. Nagoya Math. J., 56:139-145, 1974.

[11] Kjos-Hanssen, B. and Nerode, A. Effective dimension of points visited by Brownian motion. Theoretical Computer Science, 410(4-5): 347-354, 2009.

[12] Orey, S. and Taylor, S.J. How often on a Brownian path does the law of iterated logarithm fail? Proc. Lond. Math. Soc., 28(3):174-192, 1974.

[13] Potgieter, P. Nonstandard analysis, fractal properties and Brownian motion. Fractals, 17(1):117-129, 2009. 